\title{
Dynamics of the combined extra-equatorial and equatorial deep jets in the Atlantic
}

\author{
Ménesguen Claire ${ }^{1}$; Hua Bach Lien ${ }^{1, *}$; Fruman Mark D. ${ }^{1,2}$; Schopp Richard ${ }^{1}$ \\ ${ }^{1}$ Laboratoire de Physique des Océans, UMR 6523 CNRS/IFREMER/IRD/UBO-BREST, 29280 Plouzané, \\ France. \\ ${ }^{2}$ Present address: Institüt für Atmosphäre und Umwelt, Goethe Universität, Frankfurt, Germany. \\ *: Corresponding author : Hua Bach Lien, email address : lien@ifremer.fr
}

\begin{abstract}
:
The available meridional sections of zonal velocity with high vertical and meridional resolution reveal tall eastward jets at $2 \mathrm{~N}$ and $2 \mathrm{~S}$, named the extra-equatorial jets (EEJ), straddling the stacked eastward and westward jets of smaller vertical scales right at the equator, the so-called equatorial deep jets (EDJ). In contrast to the semi-annual to interannual fluctuations in the zonal velocity component, the measured meridional velocity component is dominated by intraseasonal period. We argue here that the formation mechanism for both types of jets is linked to the intraseasonal variability in meridional velocity and the associated wave motions. A process study is complemented by high resolution primitive equation simulations based on a realistic background stratification and an oscillating forcing inside the western boundary layer. The forcing confined to the upper $2500 \mathrm{~m}$ excites a spectrum of waves, including a baroclinic short Mixed Rossby-Gravity (MRG), whose instability leads to the formation of the EDJ and short barotropic Rossby waves, whose instability gives rise to the EEJ. The modeled EEJ and EDJ response is confined to the same depth range as the forcing. Potential vorticity is homogenized within specific depth ranges of westward EDJ and is found to be latitudinally confined between $2 \mathrm{~N}$ and $2 \mathrm{~S}$ by the EEJ. The combined EDJ and EEJ increase lateral mixing at the equator but also act as barriers at \pm 2 degrees of latitude.
\end{abstract}




\section{Introduction}

Observed current measurements show that equatorial regions are more energetic than midlatitudes. This is highlighted by the existence of strong, narrowly confined jets in the equatorial area of the three oceans. These highly active structures organize mixing over the whole depth of the ocean and needs therefore to be well understood.

A characteristic feature of all oceanic equatorial tracks is the vertical stacking of zonal jets alternating in direction with depth along the equator (Luyten and Swallow, 1976; Leetma and Spain, 1981; Eriksen, 1982). Their amplitudes can reach $25 \mathrm{~cm} / \mathrm{s}$ and their vertical scales are about $350 \mathrm{~m}$ in the Pacific Ocean, $500 \mathrm{~m}$ in the Atlantic and $600 \mathrm{~m}$ in the Indian (see, respectively, Firing, 1987; Gouriou et al., 1999; Dengler and Quadfasel, 2002). They have been called Equatorial Deep Jets (EDJ). Many studies have been devoted to explain their formation mechanism. For recent reviews of the literature on this topic, refer to d'Orgeville et al. (2007) (hereafter DHS07), Eden and Dengler (2008) and references therein. The mechanism proposed by DHS07 is motivated by observations showing meridional velocity field varying on intraseasonal time scales that coexists with the very slowly varying signal of the zonal jets (Weisberg and Horigan, 1981; Bunge et al., 2008). Their mechanism involves the transfer of energy from oscillating meridional motions excited by a baroclinic western boundary current to long zonal jets. Furthermore the slow variability of the jets is expressed in terms of basin modes.

A second specific system of jets is also observed in instantaneous crosssections of zonal velocity fields in the equatorial region, namely large vertical scale eastward jets straddling the EDJ and centered at \pm 2 degrees in latitude. They are observed across the entire widths of the basins with amplitudes as great as $10-15 \mathrm{~cm} / \mathrm{s}$. Gouriou et al. (2001) noticed them in their current measurements and have called them the Extra-Equatorial Jets (EEJ) (we shall use the same terminology here). They are also observed in averaged float data (Ollitrault et al., 2006) and in high resolution numerical simulations (Maximenko et al., 2005; Richards et al., 2006). In float data sets and numerical simulations, there are mul- 
tiple EEJ that alternate in direction with latitude. Nevertheless, only the eastward jets closest to the equator are strong enough to be observed in the instantaneous fields shown by Gouriou et al. (2001).

The earliest theories of EEJ formation are set in the context of either globalscale or mid-latitude dynamics of barotropic zonal jets, originally motivated by studies of the atmospheres of giant planets, where midlatitude alternating barotropic jets can be infered on instantaneous images. Rhines (1975) first evokes the arrest of the well-known turbulent $2 D$ inverse cascade of energy, due the $\beta$-effect caused by variations in the Coriolis parameter with latitude. A natural length scale arising from $\beta$ and a velocity scale $U$, is the Rhines scale $\sqrt{U / \beta}$ at which the barotropic inverse cascade is halted. Vallis and Maltrud (1993) pursue this idea, elaborating on the anisotropic character of the inverse energy cascade. Such considerations of the inverse cascade lead to a final state with an alignment of the streamlines of zonal jets along lines of constant planetary vorticity $f$. A review of recent developments in this area can be found in Thompson (2006).

Other studies focus instead on destabilization mechanisms for forming zonal jets. Indeed Gill (1974) has established that midlatitude barotropic Rossby waves can destabilize through lateral shear instability. In particular, for short enough Rossby waves (i.e. large horizontal wavenumber $K$, such that $U K^{2} / \beta$ is large, where $\mathrm{U}$ is the velocity amplitude of the Rossby wave), inertial effects dominate the $\beta$-effect and the destabilization favors the development of the fastest growing secondary wave characterized by a wavevector of similar length and orthogonal to that of the initial wave. Manfroi and Young (1999) generalized Gill's result to the case of a barotropic sinusoidal meridional flow on a $\beta$-plane, taking into account nonlinear effects, bottom drag and uniform mean flow. The previously mentioned inverse energy cascade is no longer the direct cause of zonal jets formation but can afterwards favor the merging of zonal jets. Building on those results and applying them to the equatorial case, Hua et al. (2008) (H08 hereafter) examine the destabilization of short Mixed Rossby-Gravity (MRG) waves which are known to be similar to short eastward group propagating Rossby waves.

The destabilization of short MRG waves is evidenced by H08 and DHS07 
through numerical experiments. Their equatorial basin simulations exhibit the formation of EDJ, but the excited signal in zonal velocity remains confined to the immediate vicinity of the equator: EEJ are not developped. In the present paper, our focus is both on the EEJ formation mechanism and on the potential vorticity dynamics of the joint EEJ-EDJ system. We remark that short equatorial sheared waves comprise both baroclinic short waves, such as short MRG waves, but also short barotropic Rossby waves. The destabilization of the latter has not been studied by H08 and DHS07 and we thus want to address the following question: Can the formation of the combined EEJ and EDJ system be linked to destabilization of MRG and short Rossby barotropic waves?

The paper is organized as follows: Section 2 presents the detailed spatial and temporal characteristics of the observed EDJ and EEJ, together with the intraseasonal variability in meridional velocity field. Section 3 reviews the theoretical framework for the destabilization of short intraseasonal waves leading to zonal jets. Section 4 proposes a rationalization for the dynamics of EEJ-like structures in an ocean basin, their zonal extent and their link to the amplitude and spatial characteristics of the low-frequency waves. The results are supported by various numerical simulations of the equatorial ocean. In Section 5, using a more complex and realistic simulation, we reproduce simultaneously EDJ and EEJ structures of the Atlantic Ocean and investigate the redistribution of tracers such as potential vorticity.

\section{Subsurface spatio-temporal variability of the equato- rial Atlantic}

Time-series of high vertical resolution current-meter measurements between $600 \mathrm{~m}$ and $1800 \mathrm{~m}$ depth and covering almost 7 years (2000-2006) have recently been obtained in the equatorial Atlantic at 10W and 23W (Bunge et al. (2008)). Their results corroborate and extend observations of Weisberg and Horigan (1981). The variability in the measured meridional velocity component is dominated by the 30-60 days band (Fig. 1). The observed weaker 10-15 days variability remains confined to the shallowest part of the measurements, whereas 


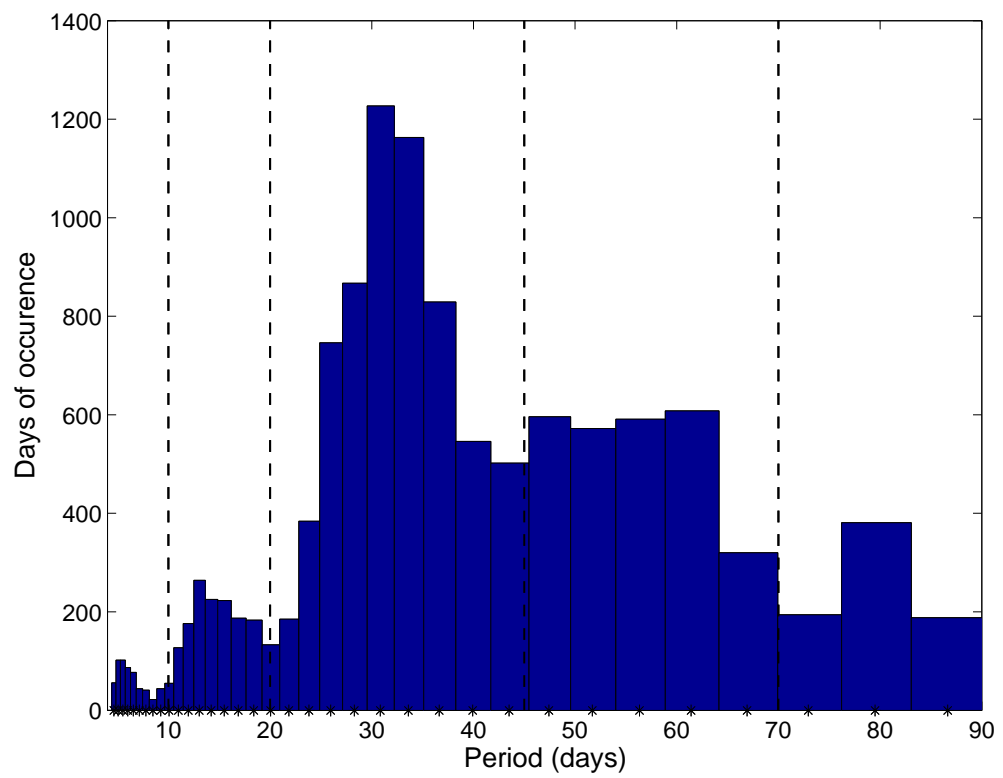

Figure 1: Distribution of the meridional velocity component of all current-meter measurements taken in the Atlantic ocean at the equator in 2000-2006 (Bunge et al., 2008)

the 30-60 days variability represents strong deep fluctuations. Oscillations can be coherent on vertical scales larger than $1000 \mathrm{~m}$ and can exceed $20 \mathrm{~cm} / \mathrm{s}$ and their spatial and temporal characteristics are generally compatible with the dynamics of free MRG waves which have a maximum in meridional velocity at the equator.

In contrast to the intraseasonal variability in the meridional velocity component, the variability in the zonal velocity component is dominated by semiannual, annual and inter-annual fluctuations, linked with dynamics specific to the equatorial region, where the observed deep zonal circulation is dominated in all three oceans by a vertical stacking of zonal jets of alternating directions along the equator. An illustration of such a circulation is given in Figure 2(a). It provides an instantaneous image of the zonal velocity component in the deep equatorial Atlantic at $23 \mathrm{~W}$, revealing alternating eastward-westward currents with a vertical scale of about $600 \mathrm{~m}$ between $1^{\circ} 30^{\prime} \mathrm{N}$ and $1^{\circ} 30^{\prime} \mathrm{S}$, the so-called EDJ. The data are taken from the EQUALANT 99 cruise (July 13 to August 21, 1999) during which several meridional transects across the equator were performed (Gouriou et al., 2001). The several meridional sections indicate that the vertical characteristic scale of EDJ is uniform across the entire basin width.

Instantaneous zonal velocity field of Figure 2(a) also reveals the existence of 


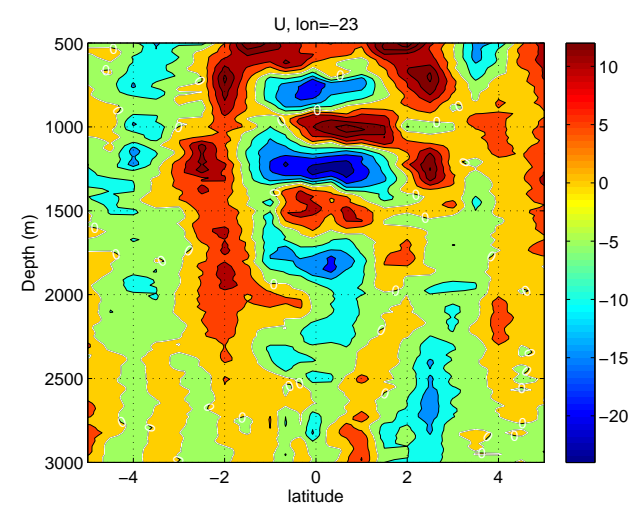

(a)

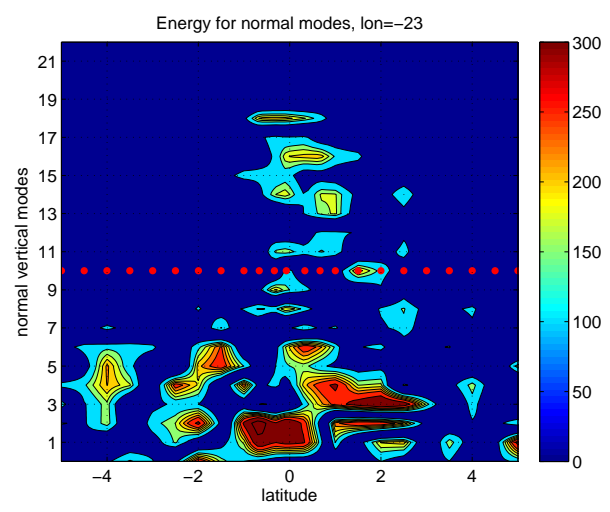

(b)

Figure 2: (a) Depth-latitude section of the zonal component of velocity in $\mathrm{cm} / \mathrm{s}$ along 23W (EQUALANT99, adapted from Gouriou et al. (2001)). Eastward (Westward) currents are positive (negative). Contour interval is $5 \mathrm{~cm} / \mathrm{s}$. (b) Energy in normal modes of zonal velocity from left panel as function of latitude and vertical mode. Red line separates high and low vertical modes. Inside the equatorial track the jets (EDJ) are highly baroclinic (the energetic modes are 16-18). At $2 \mathrm{~N}$ and $2 \mathrm{~S}$ the energy is concentrated in low modes for the EEJ.

eastward jets at $2 \mathrm{~N}$ and $2 \mathrm{~S}$ straddling the stacked eastward and westward EDJ. Similar features are readily identified in the transects at $35 \mathrm{~W}$ and $10 \mathrm{~W}$. Figure 2 of Ollitrault et al. (2006) displays the cumulated zonal velocity from float trajectories at two levels around $1000 \mathrm{~m}$ depth, confirming the existence of EEJ at $2 \mathrm{~N}$ and $2 \mathrm{~S}$, at both depths. In the float data set used by Ollitrault et al. (2006), EEJ appear as continuous, basin-wide, eastward currents with significantly stronger amplitude in the western half of the basin.

The respective vertical scales of EEJ and EDJ in the $23 \mathrm{~W}$ section are quantified by computing the vertical mode spectrum of zonal velocity as a function of latitude (Fig. 2(b)). Right at the equator, EDJ project onto vertical modes between 12 and 20, with peaks at modes 16 and 18 . Away from the equator, at $2 \mathrm{~N}$ and $2 \mathrm{~S}$, the EEJ project onto vertical modes with mode numbers smaller than 10. The two systems of zonal jets are separated by a spectral gap around mode 10 . The zonal velocity field has been high- and low-pass filtered in vertical modes for Figures 3(a) and (b) respectively. The high-pass signal represents EDJ well, while the low-pass field clearly displays the barotropic ring noted by Gouriou et al. (2001). Figure 3(b) also shows a westward flow with large vertical scale at the equator. Though the EEJ terminology originally designates the tall eastward 


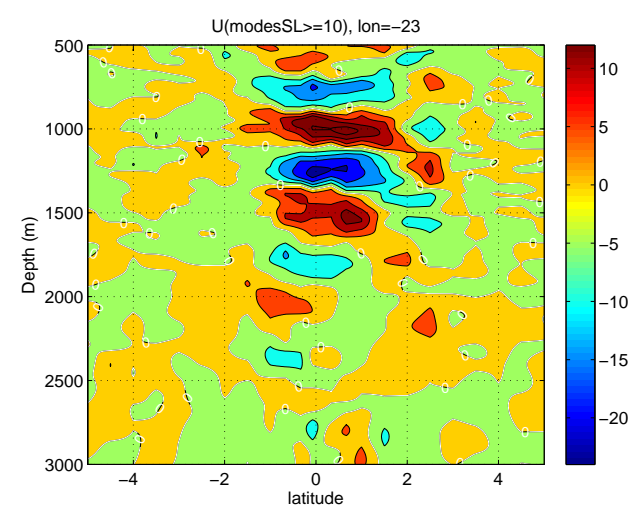

(a)

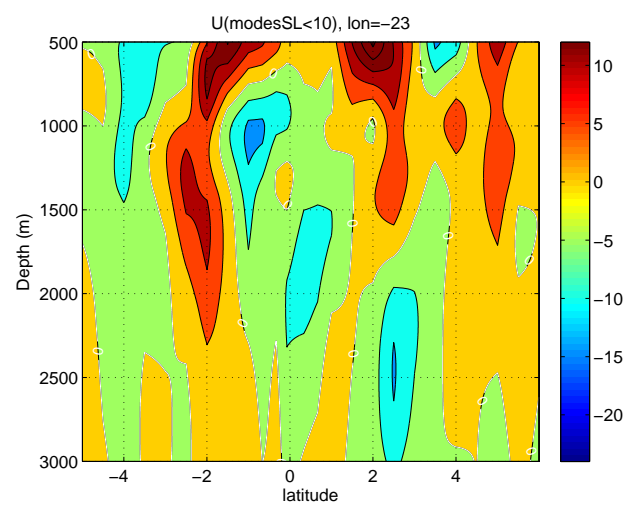

(b)

Figure 3: (a) High-pass (vertical modes $>10$ ) and (b) low-pass (vertical modes $<10$ ) filtered zonal velocity distributions corresponding to the observations shown in Figure 2. Contour interval is $5 \mathrm{~cm} / \mathrm{s}$.

flowing jets at \pm 2 degrees of latitude, the vertical projection includes the westward large vertical scale flow at the equator in the low vertical equatorial modes of the EEJ.

To summarize, equatorial measurements display two types of temporal variability: an intra-seasonal variability in meridional velocity with a period range between roughly 30 and 60 days, and a semi-annual to inter-annual variability in zonal velocity linked with the two kinds of zonal jets. The first kind, EDJ are highly baroclinic (vertical modes 12-20), basin-wide features with little zonal variation and have a meridional extension of $\pm 1^{\circ} 30^{\prime}$ about the equator. The second kind, EEJ, have a strong barotropic component and low vertical modes, also extend through the entire zonal width of the basin (but with an intensification in the western half) and their eastward flowing jets are centered at \pm 2 degrees of latitude.

\section{Zonal jets formation through lateral shear destabiliza- tion of zonally-short equatorial waves}

The present paper extends the EDJ study of DHS07 and H08 to EEJ and we thus recall their main results hereafter. 


\section{a. Basin simulations}

The numerical simulations of DHS07 use continuously stratified primitive equations to reproduce the formation of equatorial zonal jets with dynamical features comparable to those of observed EDJ. The jets are forced with a time oscillating inter-hemispheric western boundary current of large vertical scale. The oscillation excites MRG wave trains with the same vertical mode as the western boundary current. As in the realistic model of Eden and Dengler (2008), the large vertical scale MRG waves propagate into the basin interior along the equator. Their subsequent destabilization leads to EDJ-like structures with a high vertical mode number that depends only on the forcing period and not on its vertical structure. In contrast to the linear model of Kawase et al. (1992), where the equatorial circulation forced by a steady extra-equatorial source has the same vertical structure as the forcing and is intrinsically a transient spin-up adjustment, the nonlinear model of DHS07 produces a permanent jet structure displaying significantly smaller vertical scales with a basin-wide zonal extent. The spatial and temporal variabilities of these jets result from a superposition of low-frequency equatorial basin modes.

\section{b. Destabilization of short westward phase propagating $M R G$}

\section{waves}

H08 demonstrate that for short enough zonal wavelength ${ }^{1}$, the westward phase propagating MRG waves are strongly destabilized by lateral shear instability leading to the formation of zonal jets. Experiments are performed with a zonally localized MRG waves packet in a long periodic channel, allowing disturbances to propagate eastward and westward out of the perturbed unstable region. The westward group propagating part of the signal is dominated by barotropic zonal jets (EEJ-like) and the eastward group propagating part by zonal jets of small vertical scale alternating in the vertical (EDJ-like). A stability analysis of the perturbation vorticity equation reveals that, for both types of zonal jets

\footnotetext{
${ }^{1}$ the non-dimensional parameter which readily assesses the "zonally-short" character of the excited waves for each period is $k=k^{\star} \sqrt{c / \beta}$, where $k^{\star}$ is its zonal wavenumber and $c^{\star}$ the correspondant Kelvin wave phase velocity. ${ }^{\star}$ is set for dimensional parameters.
} 
(EDJ and EEJ), the meridional scale is comparable to the zonal scale of the MRG wave basic state and the growth rate is proportional to $V_{0}\left|k^{\star}\right|$, where $V_{0}$ is the meridional velocity amplitude of the basic state wave. This implies that the wave is more unstable when the zonal shear in meridional velocity is stronger. The fastest growing disturbances found in the numerical simulations have characteristics similar to those predicted by the stability analysis, most notably that the meridional scale of disturbances is of the same order as the zonal scale of the basic state wave. Since in a stratified rotating equatorial flow, vertical scale and meridional confinement about the equator are linked by the radius of deformation, there are two ways of obtaining short meridional scales: either through low meridional modes of high vertical modes or through high meridional modes of low vertical modes. These are respectively interpreted as EDJ and EEJ signals.

\section{i. High vertical modes: EDJ}

High baroclinic modes explain the vertical structure of EDJ. The vertical scale of baroclinic jets is tied to their meridional scale through the equatorial radius of deformation, evolving as the square root of the vertical wavenumber. H08 have related the vertical scale of the baroclinic zonal jets to the dominant vertical harmonic of the basic state in the fastest growing mode: $\frac{m_{\text {peak }}^{\star}}{m^{\star}} \approx 0.55 \frac{c^{\star}}{\beta} k^{\star 2} \approx$ $\frac{0.55}{4 \pi^{2}} c^{\star} \beta T^{\star 2}$, where $m^{\star}$ is the vertical wavenumber of the initial wave and $T^{\star}$ its period. $m_{\text {peak }}^{\star}$ is the vertical wavenumber of the perturbation with the fastest growth rate. Since $c^{\star}=N / m^{\star}$, the fastest growing mode, $m_{\text {peak }}^{\star}$, is independent of $m^{\star}$ and satisfies

$$
m_{\text {peak }}^{\star} \approx \frac{0.55 N \beta}{4 \pi^{2}} T^{\star 2}
$$

Shorter vertical scales of baroclinic zonal jets are favoured by shorter MRG wavelengths which have longer period.

The spatial structure of the small vertical scale zonal jets is that of a Kelvin wave, thus explaining the eastward group propagation of the high vertical mode part of the equatorial signal. 
ii. Low vertical modes: EEJ

EEJ formation occurs through the growth of low vertical mode jets of high meridional mode. The structure of the low vertical mode jets projects onto the spatial structures of long Rossby waves, explaining the predominance of low vertical modes in the westward group propagating part of the signal in the channelgeometry simulations of H08 (cf Fig.4 and Fig.5 of H08). This will guide our investigation of the dynamics of EEJ formation in a basin geometry (Section 4).

In simulations with a basin geometry and a western boundary forcing, an additional parameter must be taken into account in the analysis of the destabilization leading to westward group propagating waves. It is the product of the characteristic group velocity $c_{g}$ of the initial eastward group propagating wave forced in the western boundary with the characteristic time for the destabilization of the wave (inverse of the growth rate of the zonal jets). The larger the ratio is, the longer the distance travelled by the wave before breaking down through lateral shear instability will be. The situation is analogous to that discussed by Lacasce and Pedlosky (2004) for the destabilization of long mid-latitude Rossby waves emanating from the eastern boundary of a basin. This concept will be applied in the following Section to the case of equatorial waves with eastward group velocities forced from the western boundary. We will therefore evaluate the zonal extent of the quasi-barotropic EEJ signal resulting from the competition between the group velocity of the wave and its rate of destabilization through lateral shear instability.

\section{Assessment of forcing parameters to produce Atlantic-}

\section{like EDJ/EEJ}

Before addressing the more complex realistic case of the following section, we consider first experiments with a constant stratification $N=2 \times 10^{-3} s^{-1}$, typical of the mid-depth ocean, to assess the impact of the different characteristics of the forcing, in particular its vertical structure, strength and frequency range on the EDJ/EEJ response. 


\section{a. Impact of the forcing characteristics}

We have further investigated the vertical structure of the forcing than in the earlier studies of DHS07 and H08, where the western boundary forcing was restricted only to baroclinic mode 2 . We use the same configuration of the numerical model as in DHS07, but with a longer basin $\left(50^{\circ}\right.$ instead of $\left.30^{\circ}\right)$. The cases of a single vertical mode (either baroclinic or barotropic) forcing and one which is locally confined with depth are successively considered.

For the baroclinic case (Table 1), the oscillating forcing is imposed in the northwest and southwest corners of the basin, is antisymmetric about the equator in its meridional velocity component and is of a single baroclinic mode structure (see appendix of DHS07). This baroclinic antisymmetric forcing term mainly excites a MRG wave that branches off and propagates into the equatorial track. The characteristics of the initial wave are set by the period, the vertical mode, and the amplitude of the forcing.

\begin{tabular}{|c||c|c|c|c||c|}
\hline Exp. & $\begin{array}{c}T_{\text {forc }}(\text { days }) \\
\& \lambda=2 \pi / k^{\star}\left({ }^{\circ}\right)\end{array}$ & $V_{0}(\mathrm{~cm} / \mathrm{s})$ & $\begin{array}{c}\text { Vertical } \\
\text { mode }\end{array}$ & $\boldsymbol{m}_{\text {peak }}^{\star}$ & $\boldsymbol{L}_{\boldsymbol{D}}\left(^{\circ}\right)$ \\
\hline \hline 1 & $30 \& 7.2$ & 10 & 2 & $\mathbf{4 . 8}$ & $>\mathbf{5 0}$ \\
\hline 2 & $47 \& 4.1$ & 20 & 2 & $\mathbf{1 4 . 5}$ & $\mathbf{1 2}$ \\
\hline 3 & $50 \& 3.8$ & 20 & 2 & $\mathbf{1 6 . 7}$ & $\mathbf{1 3}$ \\
\hline \hline Exp. & $\begin{array}{c}T_{\text {forc }}(\text { days }) \\
\& \lambda=2 \pi / k^{\star}\left({ }^{\circ}\right)\end{array}$ & $V_{0}(\mathrm{~cm} / \mathrm{s})$ & $\Delta_{y}\left(^{\circ}\right)$ & $\delta_{y}\left({ }^{\circ}\right)$ & $\boldsymbol{L}_{\boldsymbol{D}}\left(^{\circ}\right)$ \\
\hline \hline 4 & $47 \& 3.85$ & 20 & 6 & 3 & $\mathbf{5 0}$ \\
\hline 5 & $50 \& 3.6$ & 20 & 3 & 1.5 & $\mathbf{2 6}$ \\
\hline 6 & $50 \& 3.6$ & 20 & 6 & 1.5 & $\mathbf{2 4}$ \\
\hline 7 & $50 \& 3.6$ & 20 & 6 & 3 & $\mathbf{2 6}$ \\
\hline 8 & $50 \& 3.6$ & 30 & 6 & 1.5 & $\mathbf{2 0}$ \\
\hline 9 & $57 \& 3.15$ & 20 & 6 & 1.5 & $\mathbf{2 1}$ \\
\hline 10 & $60 \& 3$. & 20 & 3 & 3 & $\mathbf{1 9}$ \\
\hline
\end{tabular}

Table 1: Parameters of the various experiments discussed in Section 4. Experiments 1 to 3 are forced by a signal with a baroclinic mode 2, and experiments 4 to 10 are forced by a borotropic signal. Horizontal resolution is $1 / 4^{\circ}$; and vertical resolution is between 50 to 100 levels, depending on the vertical mode expected for the EDJ. $T_{\text {forc }}$ is the period of the oscillating forcing; $\lambda$ is the zonal wavelength of the excited MRG wave; $V_{0}$ is the amplitude of the forcing; $m_{\text {peak }}^{\star}$ is the predicted baroclinic mode for EDJ-like structures; $\Delta_{y}$ and $\delta_{y}$ characterize the width of the forcing window in the barotropic forcing cases (as explained in the appendix); $L_{D}$ is the length of destabilization, evaluated as the longitude at which the eastward extra-equatorial barotropic component of zonal velocity vanishes. 
Experiments using a barotropic forcing has also been conducted in order to force barotropic waves near the equator (Table 1), since equatorially trapped waves, such as MRG waves, are purely baroclinic by construction. The forcing is moreover latitudinally confined about the equator and close to the western boundary (details can be found in the appendix). This is justified by studies of Edwards and Pedlosky (1998a) concerning the passage across the equator of water masses carried by the western boundary current. They argue that since potential vorticity of water masses crossing the equator must reverse sign, dissipation must occur in the western boundary layer at equatorial latitudes through the growth of eddies (Fig. 3 of Edwards and Pedlosky (1998a)). Our periodic meridional velocity barotropic forcing thus mimics a periodic detachment of eddies from the western boundary at the equator. The waves that propagate into the basin interior are zonally-short barotropic Rossby waves. The meridionally-confined forcing projects onto several meridional modes. The period of the excited short waves is determined by the period of the forcing, their zonal wavelengths by the Rossby wave dispersion relation, and their amplitudes by the amplitude and meridional structure of the meridional velocity of the forcing. Recapitulating, the baroclinic forcing excites a baroclinic MRG wave propagating along the equator, while the barotropic forcing excites, about the equator, a superposition of barotropic zonally-short Rossby waves with a range of meridional wavenumbers.

Figure 4 illustrates the outcome of the baroclinic or barotropic nature of the forcing. For similar parameters (50 days period oscillation and $20 \mathrm{~cm} / \mathrm{s}$ meridional velocity in the western boundary), the equatorial response set up by the destabilization of a baroclinic (resp. barotropic) forcing is shown in panels (a)(b) (resp. (c)-(d)). Let us successively examine the EDJ and EEJ responses.

(i) For both cases of baroclinic and barotropic forcing, the EDJ response, corresponding to the zonal velocity field at the equator in panels (a) and (c)), display the predominance of baroclinic mode 16-18, i.e. Atlantic-like EDJ structure. As recalled in equation (1), the vertical mode of the EDJ is a function only of the forcing period and this corresponds to a period range of about 50-60 days to produce Atlantic-like EDJ. Furthermore within that period range, short MRG waves and 


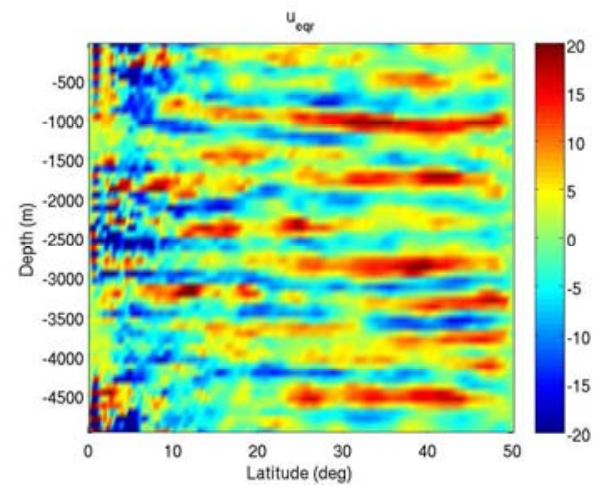

(a) Exp.3

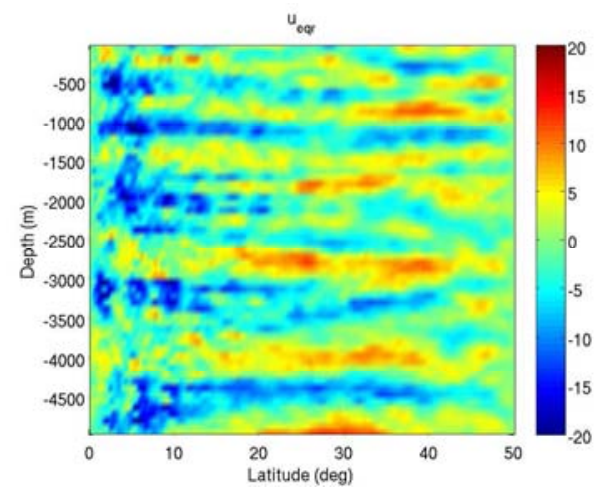

(c) Exp.7

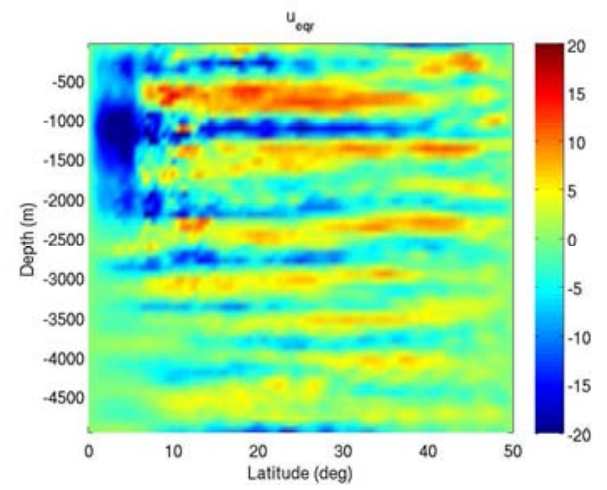

(e) Exp.11

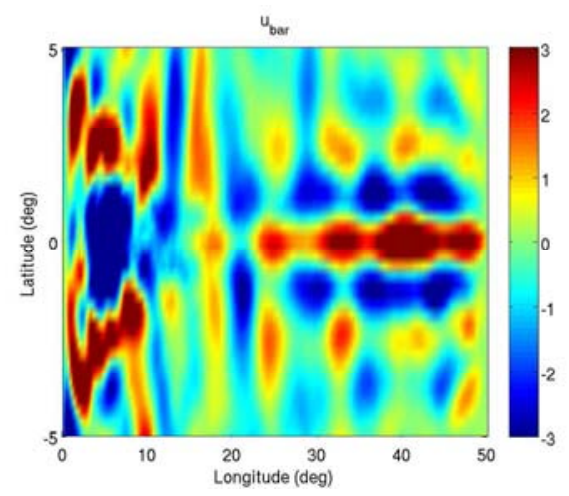

(b) Exp.3

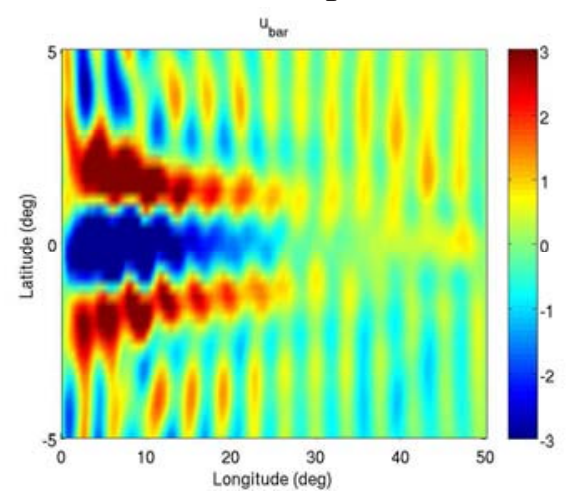

(d) Exp.7

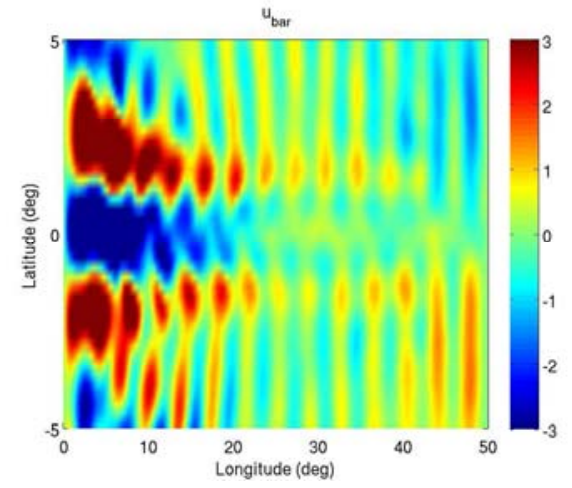

(f) Exp.11

Figure 4: Instantaneous zonal velocity distribution for cases with baroclinic forcing ((a) and (b)), a barotropic forcing ((c) and (d)) and a vertically confined forcing ((e) and (f)) after reaching a statistically steady state. The three experiments all have: a forcing period of 50 days and a forcing amplitude of $20 \mathrm{~cm} / \mathrm{s}$. (a), (c) and (e): zonal velocity section along the equator in $\mathrm{cm} / \mathrm{s}$. (b), (d) and (f): barotropic zonal velocity in $\mathrm{cm} / \mathrm{s}$. 
barotropic Rossby waves have very similar dispersion characteristics and their destabilization leads to similar EDJ characteristics. As for the amplitude of the EDJ signal, the response is found to be stronger for a baroclinic forcing.

(ii) The EEJ signal at \pm 2 degrees of latitude, seen in the barotropic zonal velocity field in panels (b) and (d), however shows an important difference between the two types of forcing: the eastward barotropic EEJ response is well developed in the barotropic forcing case and much shorter in the baroclinic case. This suggests that the barotropic component of the forcing is crucial to the formation of long EEJ-like structures.

The main goal of this work is to reproduce simultaneously EEJ-like and EDJlike structures. One possibility for exciting both barotropic and baroclinic components of the forcing is to locally confine the forcing in the vertical direction, for instance by restricting it to the upper $2500 \mathrm{~m}$ of the water column, with a vertical structure which is close to the observed vertical structure of the NADW flow in the western boundary of the equatorial Atlantic.

\begin{tabular}{|c||c|c|c|c|c|c|c|c|}
\hline Exp. & $T_{\text {forc }}$ (days) & $V_{0}(\mathrm{~cm} / \mathrm{s})$ & $N\left(\mathrm{~s}^{-1}\right)$ & $\begin{array}{c}\text { hor. res. }\left(^{\circ}\right) \\
\times \text { nb of levels }\end{array}$ & $\Delta_{y}\left(^{\circ}\right)$ & $\delta_{y}\left({ }^{\circ}\right)$ & $z_{0}(\mathrm{~m})$ & $\Delta_{z}(\mathrm{~m})$ \\
\hline \hline 11 & 50 & 50 & $2.10^{-3}$ & $1 / 4 \times 60$ & 6 & 3 & -1250 & 1000 \\
\hline 12 & $50 \& 30$ & $50 \& 30$ & $2.10^{-3}$ & $1 / 4 \times 60$ & 6 & 3 & -1250 & 1000 \\
\hline 13 & 50 & 50 & $N 23 \mathrm{~W}$ & $1 / 11 \times 400$ & 3 & 1.5 & -1250 & 1000 \\
\hline
\end{tabular}

Table 2: Parameters of the experiments discussed in Sections 4 and 5 with a "realistic" forcing. The parameters are the same as in Table 1. $N$ is the type of stratification: constant Brunt-Väisälä frequency $2.10^{-3} \mathrm{~s}^{-1}$ or the profile taken from the section at $23 \mathrm{~W}(N 23 \mathrm{~W}) . z_{0}$ and $\Delta_{z}$ characterize the localization and extension in depth of the forcing (as explained in the appendix).

The equatorial response for a vertically confined forcing case (Exp. 11 of Table 2) are shown in Figures 4 (e-f). By construction, the forcing has an intermediate vertical structure when compared to those of experiments 3 (panels (a)-(b)) and 7 (panels (c)-(d)): as expected, we observe for both the EDJ and EEJ response, intermediate characteristics for the length and strength of both types of jets. The vertical mode number, around mode 16, of the EDJ signal is set by the destabilization of a 50 days period wave. Note that the EDJ are confined at the same depth range as the oscillating forcing inside the western boundary layer. 
This seems consistent with results of Eden and Dengler (2008) who also found in their realistic GCM simulations of the equatorial Atlantic that the depth range and strength of the western boundary currents are related to the depth range and strength of EDJ-like signals. Their result is compatible with our interpretation arguing that the energy source for the EDJ is provided by the lateral shear instability mechanism present in intraseasonal wave trains excited by a forced western boundary current, whose energy propagates eastward into the basin interior. The lateral growth of the shear instability implies a mostly horizontal redistribution of kinetic energy with little vertical spreading.

\section{b. Zonal extent of EEJ}

For a purely baroclinic forcing, the regime of zonally-short baroclinic equatorial waves which is subject to lateral shear instability leads to a too short EEJ formation. In the following, we shall therefore only analyze results of simulations with a non-zero barotropic forcing. The short batrotropic Rossby waves with eastward group propagation which emanate from the western boundary forcing region have a group speed satisfying

$$
c_{g}=\beta \frac{k^{\star 2}-l^{\star 2}}{\left(k^{\star 2}+l^{\star 2}\right)^{2}},
$$

where $k^{\star}$ is the zonal wavenumber and $l^{\star}$ the meridional wave number. The characteristic time of destabilization, $\tau_{\text {destab }}$, is inversely proportional to the growth rate of the most unstable disturbances, which, from the result of Gill (1974) for barotropic Rossby waves on an infinite $\beta$-plane, is proportional to the zonal shear $V_{0} \sqrt{k^{\star 2}+l^{\star^{2}}}$.

The characteristic zonal extent of the EEJ, $L_{D}$, is estimated by the distance of the region covered by the basic state wave from the western boundary before its total destabilization

$$
L_{D} \propto c_{g} \tau_{\text {destab }}
$$

thus,

$$
L_{D} \propto L_{D}^{R} \equiv \frac{\beta}{V_{0} \sqrt{k^{\star 2}+l^{\star 2}}} \frac{k^{\star 2}-l^{\star 2}}{\left(k^{\star 2}+l^{\star 2}\right)^{2}} .
$$




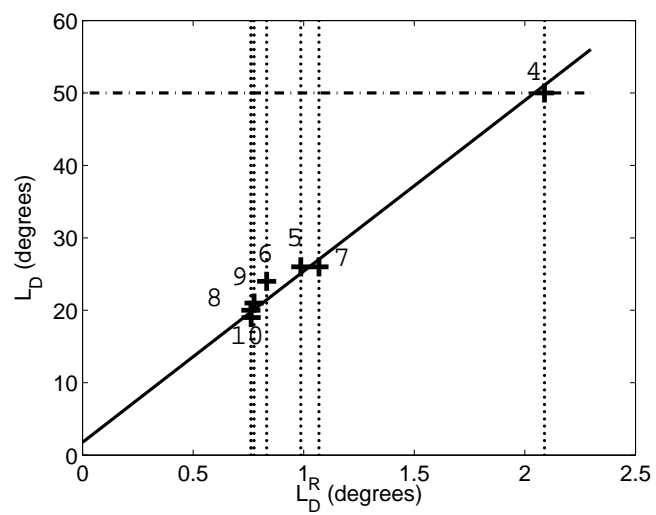

Figure 5: Longitudinal extent of wave destabilization, $L_{D}$, evaluated as the longitude at which the eastward extra-equatorial barotropic component of zonal velocity vanishes. Plotted is $L_{D}$ versus $L_{D}^{R}=\frac{\beta}{V_{0} \sqrt{k^{\star 2}+l^{\star 2}}} \frac{k^{\star 2}-l^{\star 2}}{\left(k^{\star 2}+l^{\star^{2}}\right)^{2}}$. The calculation is done on the signal averaged over 10 months. (data is in Table 1).

For a purely barotropic forcing, we expect $L_{D}$ to obey (2) and to be proportional to $L_{D}^{R}$. The parameters of the forcing are the amplitude of the meridional velocity, the period and the distribution of excited meridional modes are varied in experiments 4 to 10 (Table 1 ). We determine a meridional spectrum of forced propagating waves by applying a Fourier transform in longitude to the meridional velocity. Each zonal mode is linked through the dispersion relation to a meridional mode by the period of the forcing. $L_{D}^{R}$ is then evaluated as the predicted lengths of destabilization (equation (2)) of the most energetic zonal mode. The observed $L_{D}$, plotted against $L_{D}^{R}$ in figure 5, is the mean destabilization length, observed over a period of ten months in a statistically steady regime. This averaging removes the intrinsic variability due to the waves and, as long as the phenomenon is not dependent on basin modes, does not need to be comparable to basin-mode periods. Figure 5 shows a good agreement with the proposed proportionality law for $L_{D}$ in equation (2).

Furthermore, while the length of EEJ is inversely proportional to the forcing amplitude (equation (2)), the strength of EEJ is set by the amplitude of the forcing. In order to obtain simultaneous long EDJ/EEJ with realistic strength, the range of amplitude of the forcing is bounded by these two effects. 


\section{c. Connection with the observed Atlantic broad-band $v$ vari-}

\section{ability}

So far, we have mostly discussed simulations pertaining to a monochromatic frequency of the forcing ( 50 days period), while observations from the eastern Atlantic moorings such as those of Figure 1 indicate that the variability of the meridional velocity component presents a broad range of periods between 30 days and 60 days, with slightly stronger amplitudes near the 30 days limit. Specifically, the mean amplitude in the 25-35 days range does not exceed $8 \mathrm{~cm} / \mathrm{s}$, while the mean amplitude of the 50-60 days range does not exceed $4 \mathrm{~cm} / \mathrm{s}$ (Bunge 2008,personal communication).

In experiment 12, a bi-period forcing case is tested to assess the respective role of the two periods on the EDJ/EEJ response. The forcing takes the form of an oscillating interhemispheric western boundary current with two periods of 30 and 50 days, which is vertically confined to the upper 2500 meters (cf. Table 2 and the Appendix). The vertical confinement of the boundary current implies a nonzero barotropic component of the forcing, needed to force long EEJ, as discussed in the previous paragraph and both barotropic Rossby waves and baroclinic MRG waves are excited in the equatorial area. The amplitudes of the forcing near the western boundary forcing are listed in Table 3 for each period and each vertical mode. The non-dimensional parameter $k$, which characterize the zonal-shortness character of the excited waves, is $k=-2$ and -3.9 for the 30 and 50 days period forcing for, for instance, vertical mode 2. According to H08 results, the 30 days forcing is borderline for exciting zonally-short waves, and one should not expect significant destabilization for that component. Furthermore, when looking at the components of the 30 days forcing of experiment 12 , none of the vertical modes has an amplitude which exceeds $10 \mathrm{~cm} / \mathrm{s}$. All excited waves are thus more stable than the 30 days wave excited in experiment 1 . Figures 6(a-b) illustrate the equatorial response for experiment 1 and show that only very weak EEJ are formed with no EDJ at all. We conclude that low amplitude 30 days period waves are quasi-stable in experiment 12 .

For the bi-period, vertically confined experiment (Exp. 12), Figures 6 (c-d) 


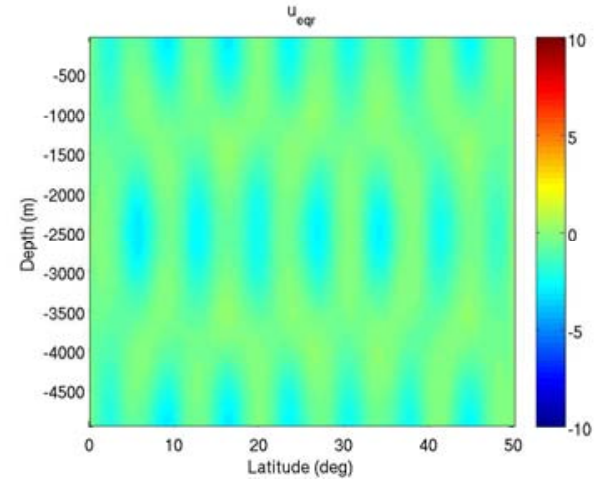

(a) Exp.1

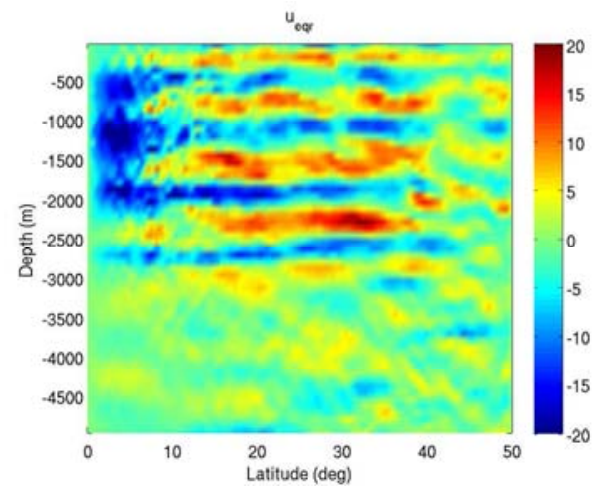

(c) Exp.12

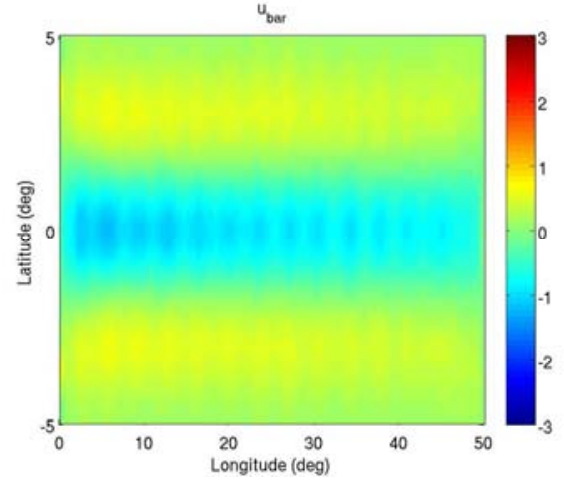

(b) Exp.1

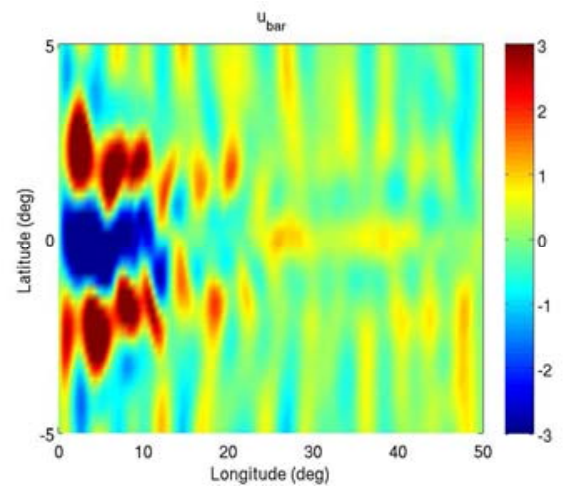

(d) Exp.12

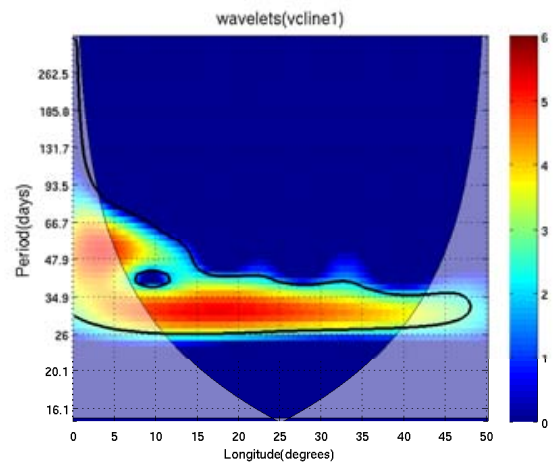

(e) Exp.12

Figure 6: (a-b) Instantaneous zonal velocity distribution for a forcing period of 30 days and a forcing amplitude of $10 \mathrm{~cm} / \mathrm{s}$ with a baroclinic mode 2 (Exp. 1) after reaching a statistically steady state. (a) zonal velocity section along the equator in $\mathrm{cm} / \mathrm{s}$. (b) barotropic zonal flow in $\mathrm{cm} / \mathrm{s}$. (c-d) As in (a-b) but for a forcing with two periods (30 days and 50 days) in the western boundary and with a vertical confinement and a latitudinal confinement about the equator (Exp. 12). (e) Wavelet analysis of the first baroclinic mode of meridional velocity at the equator from the two-frequency forcing case. The color field is for $v$ amplitude $(\mathrm{cm} / \mathrm{s})$. The 50 days signal remains confined to the western boundary while the 30 days signal is still strongly energetic over the entire basin width. 
displays the equatorial response. Panel (c) gives evidence of an EDJ system with a high vertical mode around mode 16, while panel (d) displays EEJ length which is similar to the value observed in Figure 4 (f). It therefore appears that the total response for a bi-period forcing case is dominated by the the 50 days period component of the forcing: the only wave which is destabilized is the 50 days wave while the 30 days wave is quasi-stable. This is furthermore corroborated by the wavelet analysis of the variability of the first baroclinic mode of the meridional velocity component $v$ as a function of longitude (Fig. 6 (e)). The 50 days signal remains confined to the western boundary since its quick destabilisation leads to quite smaller amplitudes of the 50 days signal further eastward. On the other hand, the 30 days signal remains constant throughout the whole basin width. The same analysis for the second main vertical component of $v$, the barotropic component, has been done (not shown) with similar conclusions. This is thus consistent with observations of Bunge et al. (2008) which are restricted to the eastern half of the Atlantic basin and which indicate that meridional velocity is the most energetic in the 20 to 45 days band since those periods with observed amplitudes are quasi stable to lateral shear instability throughout the whole basin.

\section{Atlantic case}

\section{a. EDJ/EEJ for a realistic stratification and forcing}

Our previous simulations as well as those of DHS07 and H08 are initialized with a constant Brunt-Väisälä frequency $N$. In order to better reproduce the observed Atlantic fields, we use a depth-varying Brunt-Väisälä frequency $N(z)$ typical of the central equatorial Atlantic ocean, corresponding to the meridionallyaveraged stratification of the EQUALANT99 section taken along 23W (Fig. 7(a)).

The forcing takes the form of an oscillating interhemispheric western boundary current with a monochromatic period of 50 days, that is vertically confined to the upper 2500 meters, a forcing consistent with the observed dynamics of the western equatorial Atlantic. The parameters of this "realistic" experiment are listed in Table 2 (Fig. 7(b)) and details are given in the Appendix. Both short barotropic Rossby waves and short baroclinic MRG waves are excited. Table 3 


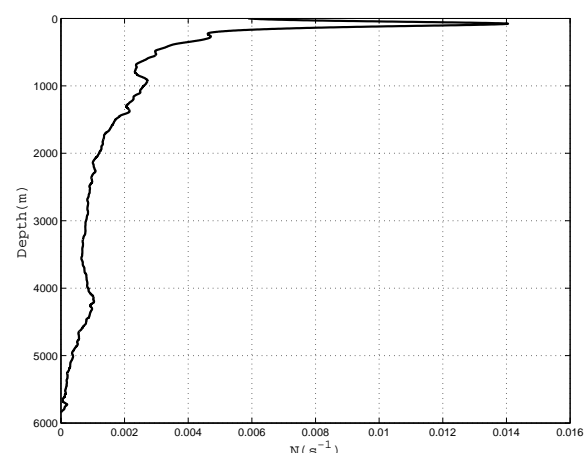

(a)

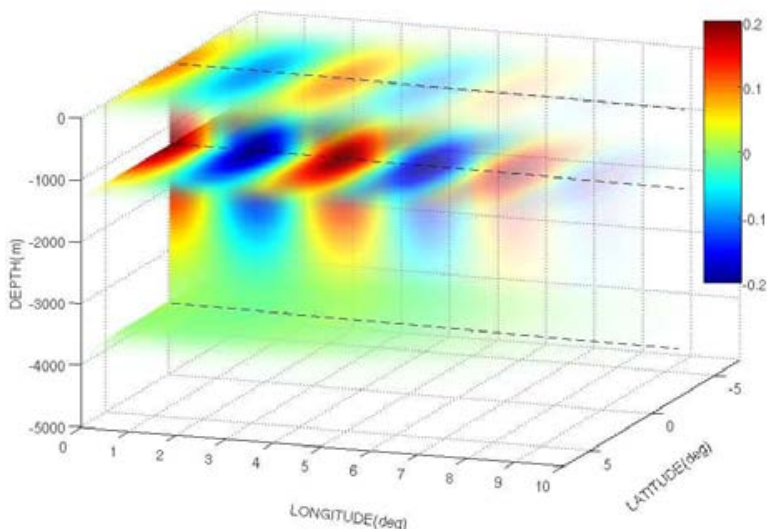

(b)

Figure 7: (a): Meridionally averaged vertical Brunt-Väisälä frequency profile for the 23W section of EQUALANT99, used in the simulations of the Atlantic equatorial jets. (b): Spatial structure of the meridional velocity used to force the system at the equator in the western boundary.

shows the projection of the forcing onto the lowest four vertical modes, the predicted zonal length $L_{D}$ of the EEJ and the dominant vertical mode of the EDJ for each vertical mode of the forcing.

\begin{tabular}{|c||c||c|c|c|c|}
\hline & Vertical mode & 0 & 1 & 2 & 3 \\
\hline \hline$T_{\text {forc }}=30$ days & amplitude $(\mathrm{cm} / \mathrm{s})$ & 9.8 & 7.5 & 1.2 & 2.7 \\
\hline Exp.12 & $m_{\text {peak }}^{\star}$ & -- & 5.6 & 4.7 & 3.8 \\
\hline & Predicted $L_{D}\left(^{\circ}\right)$ & $>70$ & 70 & 530 & 330 \\
\hline \hline$T_{\text {forc }}=50$ days & amplitude $(\mathrm{cm} / \mathrm{s})$ & 16.3 & 12.4 & 2.0 & 4.6 \\
\hline Exp.12 & $m_{\text {peak }}^{\star}$ & -- & 17.7 & 16.7 & 15.7 \\
\hline & Predicted $L_{D}\left(^{\circ}\right)$ & $>30$ & 10 & 47 & 27 \\
\hline \hline$T_{\text {forc }}=50$ days & amplitude $(\mathrm{cm} / \mathrm{s})$ & 17.8 & 8.3 & 9.5 & 7.4 \\
\hline Exp.13 & $m_{\text {peak }}^{\star}$ & $X X$ & 17.7 & 16.7 & 15.7 \\
\hline & Predicted $L_{D}\left(^{\circ}\right)$ & $>30$ & 13 & 14 & 17 \\
\hline
\end{tabular}

Table 3: Characteristics of the barotropic mode and the first 3 baroclinic normal modes of the experiments using realistic stratification: their amplitudes, the dominant baroclinic vertical mode they excite through barotropic instability $\left(m_{\text {peak }}^{\star}\right)$, and the length of destabilization for each mode according to the inferred proportionality laws.

The characteristics of the confined forcing are:

(i) that it contains many vertical modes, in particular a barotropic component, favoring the formation of long EEJ-like structures [the resulting $L_{D}$ for this combined vertical modes forcing is estimated in the simulation to be of $18.7^{\circ}$, in agreement with the result shown in Figure 8(c)]. 
(ii) that it is composed of multiple baroclinic modes oscillating with the same time-period, leading to the formation of a single EDJ-like structure [since excited short equatorial waves of the different baroclinic modes have all the same period, imposed by the western boundary forcing term, they are destabilized into EDJ with nearly the same vertical scale].

Figure 8(a) shows a longitude-depth plot of the instantaneous zonal velocity field in the model along the equator once the kinetic energy has reached its equilibrium value. Again, note that the EDJ are confined at the same depth range as the oscillating forcing inside the western boundary layer, creating jets as energetic as the forcing was (e.g. the amplitude of the jets is the same as that of the meridional velocity oscillation in the western boundary).

Figure 9(a) shows an instantaneous meridional section of zonal velocity amplitudes taken a distance of $10^{\circ}$ from the western boundary. It displays the same overall vertical patterns as the ones seen in the observed instantaneous section at 23W (see Figure 2(a)), with EDJ and EEJ reaching comparable amplitudes. Both Figures 8(a) and 9(a) reveal an increase in the vertical scales of the EDJ with depth, due to the depth variation of the realistic stratification used.

The projection of the zonal velocity of Figure 9(a) onto vertical modes corresponding to $N(z)$ (Fig. 9(b)) reveals that a forcing period of 50 days excites EDJ with a peak at vertical mode 18 (vertical wavelength $\approx 600 \mathrm{~m}$ ) consistent with the observed in situ data (Fig. 2(b)). The meridional sections of high- and low-pass filtered zonal velocity fields are displayed in Figures 10(a) and 10(b). They bear a high resemblance with observations of Figure 3 and, this, despite the relatively simple monochromatic nature of the forcing in the numerical simulation.

Figure 10(b) shows an instantaneous barotropic zonal velocity field after the model has reached a statistically steady state. Eastward zonal jets are well reproduced and centered at about $2 \mathrm{~N}$ and $2 \mathrm{~S}$ resembling the observed EEJ. We want to stress here again that even if Figure 2 of Ollitrault et al. (2006) contains cumulated Lagrangian floats velocities and provides thus an average rather than instantaneous view of the zonal velocity field, continuous eastward jets are clearly 


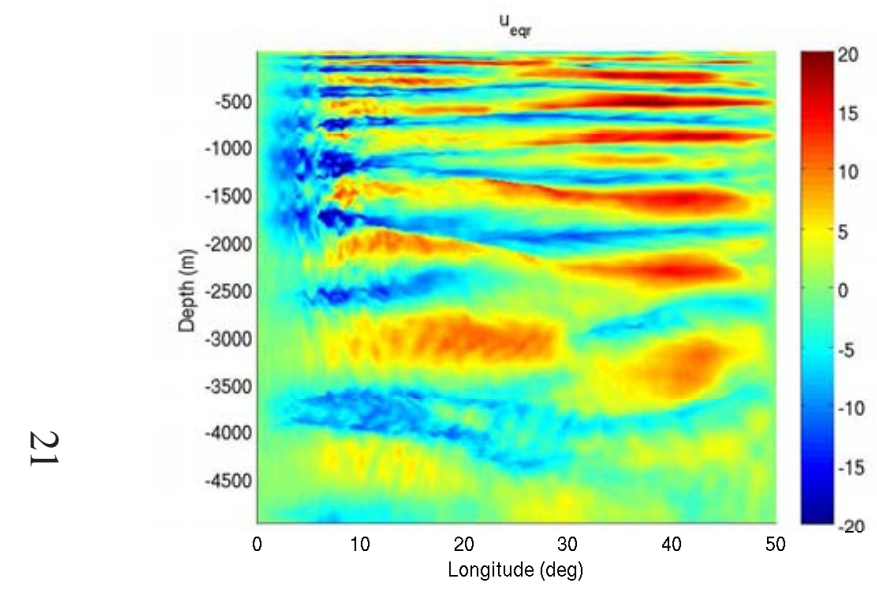

(a)

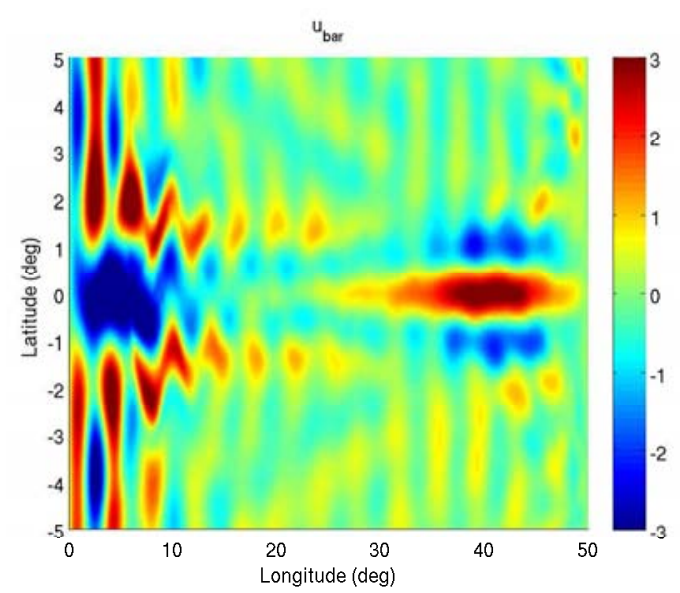

(b)

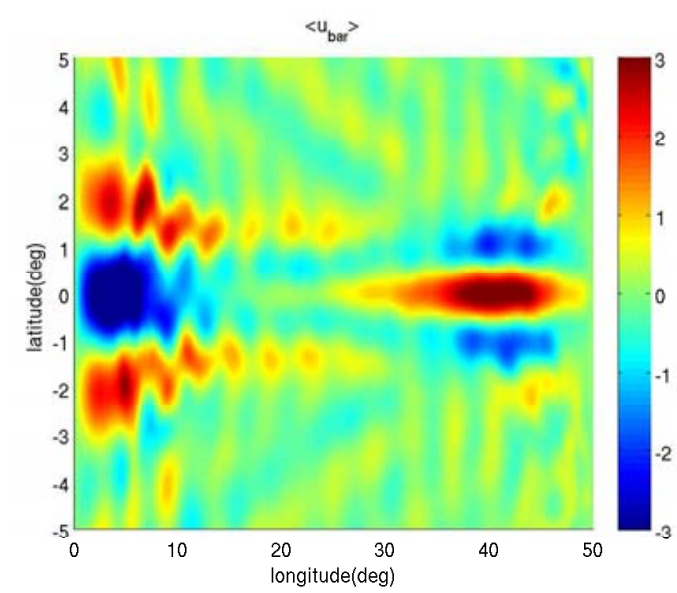

(c)

Figure 8: Zonal velocity distribution in Atlantic case experiment (Exp.13) after reaching a statistically steady state. (a) Instantaneous zonal velocity along the equator in cm/s. (b) Instantaneous barotropic component in cm/s. (c) Barotropic component in cm/s, time-averaged (over 290 days, using 10 snapshots). The main features and characteristics of the jets in the equatorial track are reproduced in the simulation: amplitude, vertical scale, meridional location of EEJ. This figure can be compared to observation in Figure 2. 


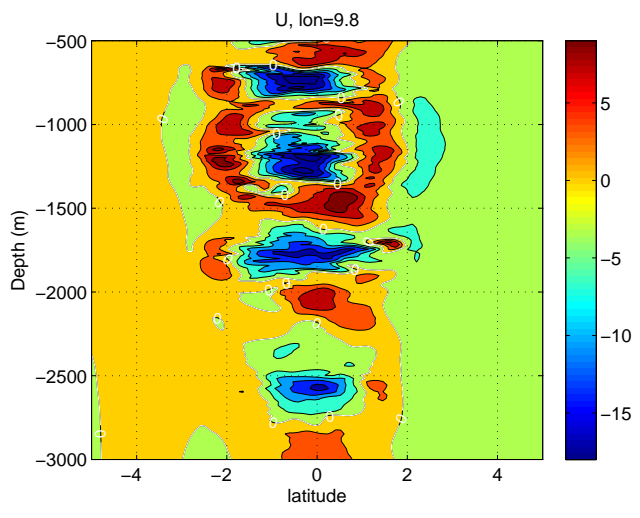

(a)

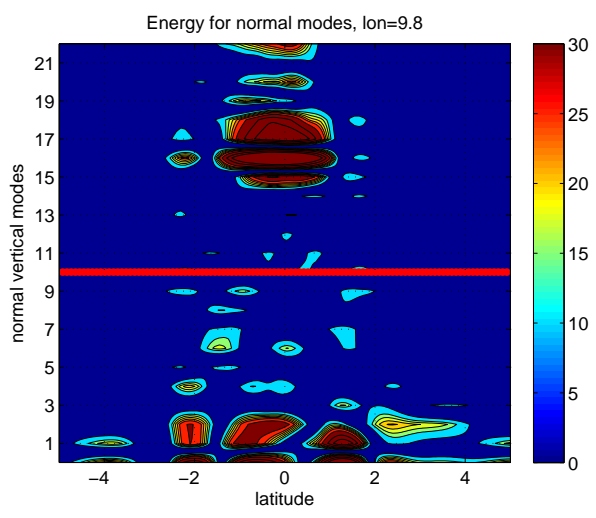

(b)

Figure 9: (a) Meridional section of zonal velocity at $9.8^{\circ}$ from the western boundary. (black contour every $3.5 \mathrm{~cm} / \mathrm{s}$ ). (b) Energy in zonal velocity as a function of normal mode from the field shown in the left panel. Red line separates high and low vertical modes.

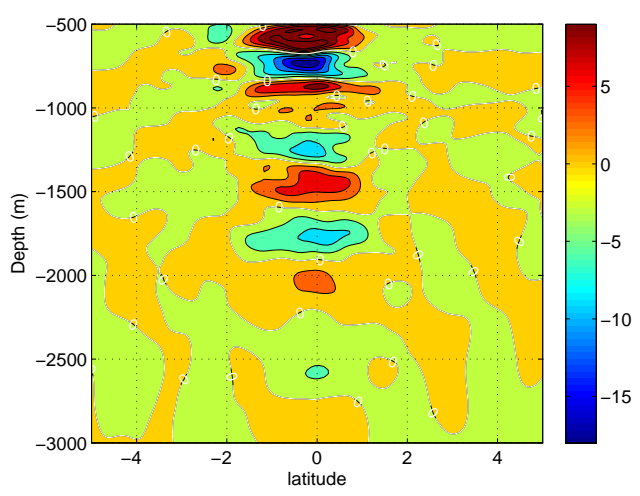

(a)

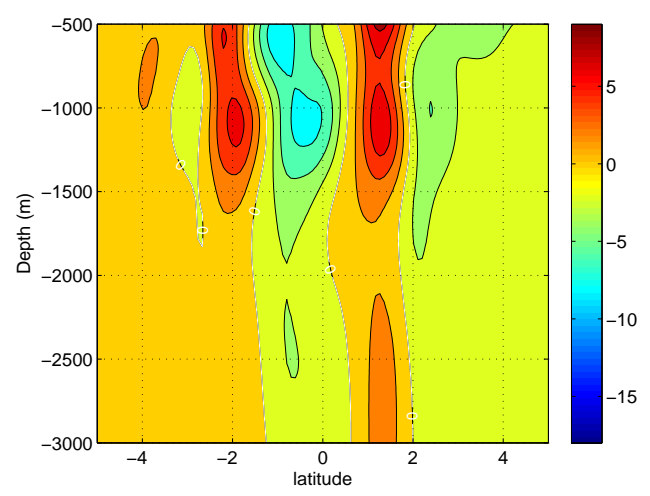

(b)

Figure 10: (a) High-pass (vertical modes > 10) and (b) low-pass (vertical modes < 10) filtered zonal velocity distribution corresponding to the field of Figure 9. Contour interval is $3 \mathrm{~cm} / \mathrm{s}$ for high modes and $2 \mathrm{~cm} / \mathrm{s}$ for low modes. High and low pass filters respectively display the EDJ and the EEJ. This figure should be compared to the observations shown in Figure 3. 
identifiable in the instantaneous velocity field (Gouriou et al. (2001)) and highlight their considerable strength when compared to midlatitude zonal jets whose signal requires a long time averaging to become visible. This last point is correctly reproduced by the Atlantic simulation (Exp. 13).

\section{b. Potential vorticity distribution}

Observed distributions of potential vorticity and passive tracers display a strong meridional homogenization within specific depth ranges near the equator at depth (see, e.g., d'Orgeville et al. (2004) for the equatorial Atlantic, and Ménesguen et al. (2009)). We have diagnosed Ertel potential vorticity in experiment 13, using $\mathrm{PV}=(2 \boldsymbol{\Omega}+\nabla \wedge \boldsymbol{u}) \cdot \nabla \rho / \rho_{0}$, which is a conserved quantity along a fluid parcel trajectory in the absence of viscosity and external forcing, where $\Omega$ is the Earth rotation vector, $\boldsymbol{u}$ is the fluid parcel's velocity, $\rho$ its density, and $\rho_{0}$ is a constant reference density.

PV is diagnosed along isopycnal surfaces located within westward and eastwardflowing EDJ, respectively, and is displayed in Figures 11(a) and 11(b). There are striking differences in the latitudinal distribution of PV between the two cases. Within westward EDJ, PV is completely homogenized to a value close to zero between $2 \mathrm{~N}$ and $2 \mathrm{~S}$ where sharp jumps in PV appear, corresponding to temporalmean positions of EEJ (Fig. 8(b)). In contrast, at depths of eastward-flowing EDJ, right at the equator, PV displays a strong meridional gradient, steeper than the one of planetary vorticity, with narrow meridional plateaus of less than $1^{\circ}$ latitude in width observed further away from the equator. These two types of PV distribution, typical of finite-amplitude westward and eastward flows centered at the equator, are also found in channel geometry simulations by Fruman et al. (2009), who show an equilibration of zonal jets through inertial instability redistributing angular momentum meridionally and mixing PV to near zero values. Ménesguen et al. (2009) argue how a flatened PV gradient can be interpreted as being due to local PV mixing by inertial instability rather than simply the passage of a Rossby wave.

The vertical distribution of homogenized PV patterns in the Atlantic simu- 


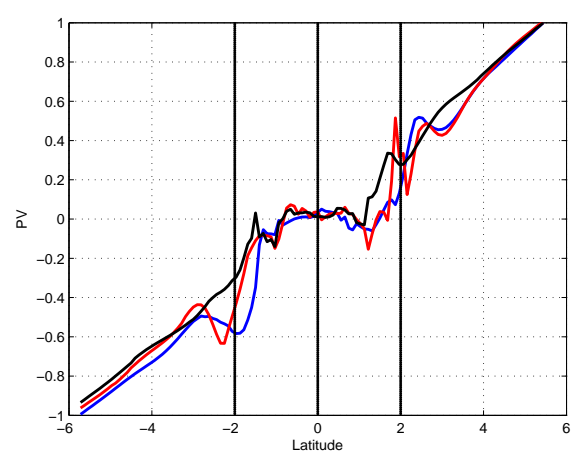

(a)

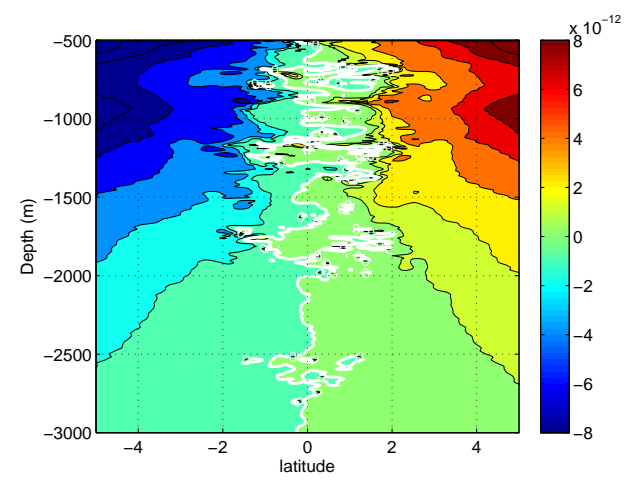

(c)

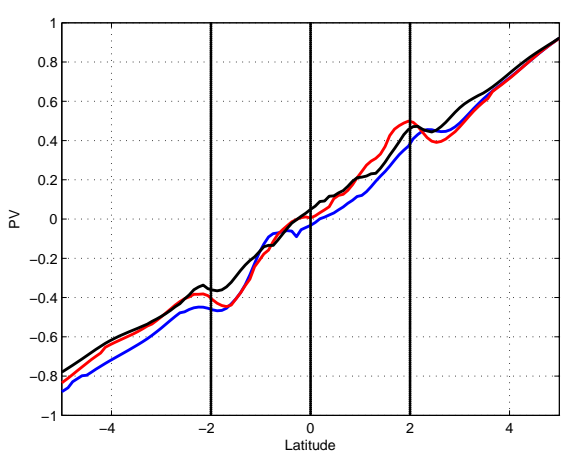

(b)

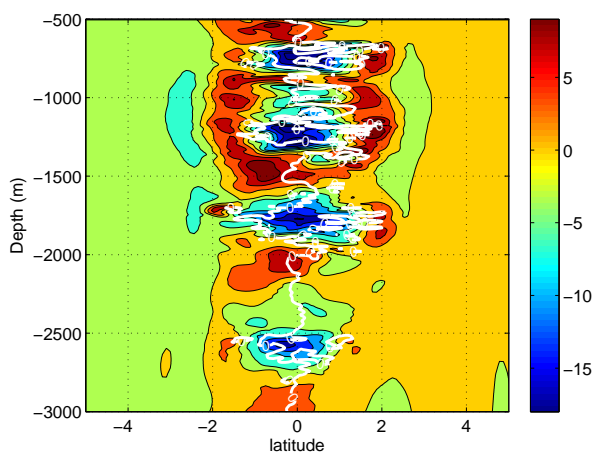

(d)

Figure 11: (a) Normalized potential vorticity versus latitude profiles on isopycnal surfaces at levels: $z \sim 790 \mathrm{~m}, z \sim 1190 \mathrm{~m}$ and $z \sim 2540 \mathrm{~m}$ (all inside westward jets), and (b) at levels: $z \sim 580 \mathrm{~m}, z \sim 1460 \mathrm{~m}$ and $z \sim 2080 \mathrm{~m}$ (inside eastward jets). (c) Meridional section of potential vorticity at $7.5^{\circ}$ from the western boundary, with zeroPV isoline in white. (d) Meridional section of zonal velocity at $9.8^{\circ}$ from the western boundary (black contours every $3.5 \mathrm{~cm} / \mathrm{s}$, with Zero-PV isoline in white). Westward jets exhibit lateral homogenization of tracers with potential vorticity mixed to zero. 
lations can be assessed from Figure 11(c). Four main weak PV regions of large latitudinal extent can be identified by the zero PV isoline (white contours) that has been overlaid on the latitude-depth distribution of zonal velocity. There is clearly a higher concentration of wide meridional excursions of the zero-PV contours within the depth ranges of westward EDJ. Furthermore, the latitudinal confinement of zero-PV regions by the taller eastward EEJ at $2 \mathrm{~N}$ and $2 \mathrm{~S}$ is observed throughout the water column. Quasi-barotropic eastward zonal jets are known to act as barriers to meridional fluid displacements. A review and rationale for this property of mid-latitude zonal jets can be found in Dritschel and McIntyre (2008).

Muench et al. (1994) were the first to diagnose potential vorticity in the subsurface equatorial dynamics from observations in the equatorial Pacific and found that the presence of EDJ induces significant departures of the high-pass filtered PV field from the planetary vorticity contribution. They concluded that EDJ could not be interpreted as linear Kelvin waves since the latter do not carry PV. Instead, the observed EDJ meridonal structure matches that of the first meridional mode Rossby wave, although the zero crossings in zonal velocity are at $1^{\circ} 30^{\prime} \mathrm{N}$ and $1^{\circ} 30^{\prime} \mathrm{S}$, rather than the $1 \mathrm{~N}$ and $1 \mathrm{~S}$ predicted by linear wave theory. For the equatorial Atlantic data set, Johnson and Zhang (2003) also identify spatial scales of EDJ with a first meridional mode Rossby wave, again noting that the meridional extension of the EDJ is wider than predicted by linear wave theory. For the equatorial basin simulations of DHS07 using an oscillating forcing in the Western boundary layer, the EDJ formed are found to be the components of basin modes, thus involving finite-amplitude, long Kelvin and Rossby waves of high vertical modes. The corresponding potential vorticity field displays a slight homogenization at westward jet levels but the simulated EEJ are rather weak and remain confined to the westernmost part of the basin.

In the present simulations, the modelled EEJ reach amplitudes comparable to those of the observed EDJ, explaining why EEJ have a strong quantitative influence on the meridional structure of the PV field (Fig. 11(a,c)). Thus, a possible interpretation of the wider meridional scales noted by Muench et al. (1994) and Johnson and Zhang (2003) is to attribute them to the low-vertical EEJ mode rather 
than to the high-vertical EDJ mode, since taller structures correspond to larger radii of deformation than shorter structures. Quantitatively, the radii of deformation for vertical modes $16-18$, with which we associate the EDJ, are between $0.79^{\circ}$ and $0.84^{\circ}$, while for vertical modes 1 and 2 , the radii of deformation are $2.9^{\circ}$ and $2.1^{\circ}$. For the lowest meridional mode equatorial Rossby wave, the latitude of zero zonal velocity coincides with the deformation radius. The observed zero crossings are thus close to those of the equatorial Rossby waves with low vertical modes, and we thus attribute the structure of the EEJ with them. In our simulations at least, the total PV distribution results from nonlinear scale interactions between the quasi-barotropic EEJ and the 600 m-vertical-scale EDJ and is not a simple superposition of the respective contributions of the low-pass and high-pass filtered fields.

\section{Discussion}

In this study, based on equatorial wave instability (H08) and using a highresolution primitive equations model of an equatorial ocean basin, we have shown how waves excited by intra-seasonal variability of meridional velocity in the deep ocean destabilize and generate zonal jets.

The EEJ signal is associated with growing perturbations whose energy propagates westward from regions where destabilization occurs. In an ocean basin forced in the western boundary layer, to induce growth of EEJ structures extending far into the basin interior is therefore not obvious. We have defined the zonal extent $L_{D}$ of the EEJ structure to be the distance covered by unstable waves. We showed that in a purely barotropic forcing, $L_{D}$ depends on the amplitude, the meridional wave length and the zonal scale of the unstable wave. A first result is that the longer and weaker the forced wave is, the further away from the western boundary it will propagate. A second result is that for the same amplitude, a barotropic wave propagates further into the basin, before being totally destabi-

lized, than a baroclinic wave meaning that a barotropic forcing will favor long EEJ-like structures.

In order to produce EEJ and EDJ in an Atlantic typical stratification, the 
model was driven by meridional oscillating western boundary forcing confined to the upper $2500 \mathrm{~m}$ depth extending several degrees about the equator and mimicking the observed destabilization through periodic eddy formation of the western boundary current while it crosses the equator. This particular configuration excites a short barotropic Rossby wave together with equatorially confined baroclinic Rossby and MRG waves propagating into the basin interior. The barotropic wave exhibits a greater length of destabilization than the baroclinic equatorially trapped modes oscillating with the same period. Such forcing, with a single period of 50 days but composed of several vertical and meridional modes, can be tuned to simultaneously form EDJ of vertical mode 18 (the $\sim 600 \mathrm{~m}$ vertical scale observed in the Atlantic) together with long barotropic EEJ. It also reproduces the intensification of the observed signal in the upper $2500 \mathrm{~m}$ and the western intensification of the EEJ seen in the Atlantic observations of Ollitrault et al. (2006).

Even if it contains the ingredients necessary to produce the complex dynamics in the equatorial track, this forcing remains nevertheless academic. It could be made more realistic by including the intrinsic destabilization of the western boundary current. In that case, the eddying area of Edwards and Pedlosky (1998b) is no longer confined to the first few degrees of the western boundary but penetrates further eastward into the equatorial track. The wider zonal extent of the forcing facilitates formation of EEJ over the whole width of the basin. The eddying area spreads also further away from the equator and excites in a wider latitudinal window short barotropic Rossby waves. Since the amplitude of the interior jets is directly tied to the western boundary forcing, a wide latitudinal eddying area, whose strength decays from the equator, produces weak zonal extraequatorial jets poleward EEJ (not shown). A more realistic forcing would also include a downward propagation of energy from surface instabilities. When energy propagates at depth in a form of a zonally short wave, lateral shear instability is a good candidate to cause the breaking of the wave leading to the creation of equatorial zonal jets.

Combined to the specific zonal velocity structure in the equatorial track are fine homogenized density layers localized inside westward EDJ and between the 
EEJ (d'Orgeville et al., 2004). The numerical model shows that potential vorticity is well homogenized laterally only if it reproduces the combined system of jets: EDJ and EEJ (not shown). Such tracer redistribution is a strong signature of a high dynamical mixing, occuring in the equatorial track. The EEJ barriers prevent meridional redistribution of tracers. We have found that they increase the meridional gradients of potential vorticity at \pm 2 degrees, isolating meridionally the layers of well-mixed PV appearing within westward EDJ. The process of PV mixing is further investigated in Ménesguen et al. (2009).

Finally, the present work has been mostly motivated by equatorial Atlantic observations which have guided our model setup and our limited parameter space exploration. However EEJ are also observed in the equatorial Pacific ocean, and their specific case is addressed by Ascani et al. (2008).

Acknowledgments. This work has benefited from grant 81777 from IDRIS. Access to the Earth Simulator is through IFREMER-CNRS-ES MOU. We would like to thank S. Le Gentil for her development in ROMS. Support from the French ANR (Agence Nationale pour la Recherche), contract no. ANR 05 CIGC 010 is acknowledged. The authors gratefully acknowledge the insightful comments of the anonymous reviewers. 


\section{Appendix}

The forcing confined to the western boundary and about the equator is introduced in the model as follows.

The coordinates are such that $x, y, z$ denote respectively the zonal, meridional, vertical coordinates with $x=0$ at the western boundary, $y=0$ at the equator and $z=0$ at the see surface.

A smooth boxcar function is applied to confine the forcing to the western boundary and to the equatorial region (see Fig. 7(b)). Within this boxcar, the flow is restored to the forcing with a time restoring scale of $t_{0}=2$ days. The restoring term $\sigma$ is written as

$\left.\left.\left.\left.\sigma=1 / t_{0} * 0.5\left(\tanh \left[\left(x+x_{0}\right) / \delta_{x}\right)\right]-\tanh \left[\left(x-x_{0}\right) / \delta_{x}\right)\right]\right) * 0.5\left(\tanh \left[\left(y+y_{0}\right) / \delta_{y}\right)\right]-\tanh \left[\left(y-y_{0}\right) / \delta_{y}\right)\right]\right)$,

with $x_{0}=y_{0}=4.5^{\circ}$ and $\delta_{x}=3^{\circ} . \delta_{y}$ values are found in Tables 1 and 2. The forcing structure is

$$
\tilde{v}=V(x, y, z) \cos (\omega t),
$$

with $V(x, y, z)=V_{0} * G(x) F(y) H(z)$.

The zonal structure is defined as

$$
G(x)=\cos \left(k^{\star} x\right),
$$

with $k^{\star}$ given by the dispersion relation of the wave to be forced (MRG or barotropic Rossby waves).

The meridional structure is defined as

$$
F(y)=\exp \left(-0.5\left(y / \Delta_{y}\right)^{2}\right)
$$

where $\Delta_{y}$ is given in Tables 1 and 2 for experiments $4-13$. The forcing is centered at the equator.

The vertical structure is defined as

$$
H(z)=\cos \left(m^{\star} z\right)
$$


where $m^{\star}$ is the prescribed forcing vertical mode for purely baroclinic forcing (Exp.1-3, Table 1);

$$
H(z)=1
$$

for barotropic forcing (Exp.4-10, Table 1); or

$$
H(z)=\exp \left(-0.5\left(\left(z-z_{0}\right) / \Delta_{z}\right)^{2}\right)
$$

where $z_{0}$ and $\Delta_{z}$ are given in Table 2 for experiments $11-13$. The total depth is $h_{0}=5000 \mathrm{~m}$. The forcing term is confined at the surface. It is a superposition a strong barotropic component and several baroclinic modes (Table 3). 


\section{REFERENCES}

Ascani, F., E. Firing, and P. McCreary, J. P.and Dutrieux. 2008. Deep mean zonal equatorial currents generated by Yanai wave beam. In Ocean Sciences Meeting, Orlando.

Bunge, L., C. Provost, B. L. Hua, and A. Kartavtseff. 2008. Variability at intermediate depths at the equator in the Atlantic ocean on 2000 - 2006: annual cycle and equatorial deep jet. J. Phys. Oceanogr.

Dengler, M. and D. Quadfasel. 2002. Equatorial deep jets and abyssal mixing in the indian ocean. J. Phys. Oceanogr., 32, 1165-1180.

d'Orgeville, M., B. Hua, R. Schopp, and L. Bunge. 2004. Extended deep equatorial layering as a possible imprint of inertial instability. Geophys. Res. Letts., 31.

d'Orgeville, M., B. Hua, and H. Sasaki. 2007. Equatorial deep jets triggered by a large vertical scale variability within the western boundary layer. J. Mar. Res., $65,1-25$.

Dritschel, D. and M. McIntyre. 2008. Multiple jets as PV staircases: the phillips effect and the resilience of eddy-transport barriers. J. Atmos. Sci. in press.

Eden, C. and M. Dengler. 2008. Stacked jets in the deep equatorial Atlantic ocean. J. Geophys. Res., 113(C04003).

Edwards, C., A. and J. Pedlosky. 1998a. Dynamics of nonlinear cross-equatorial flow. part 1: Potential vorticity transformation. J. Phys. Oceanogr., 28, 23822406.

Edwards, C. and J. Pedlosky. 1998b. Dynamics of nonlinear cross-equatorial flow. part 2: The tropically enhanced instability of the western boundary current. J. Phys. Oceanogr., 28, 2406-2417.

Eriksen, C. 1982. Geostrophic equatorial deep jets. J. Mar. Res., 40, 143-157. 
Firing, E. 1987. Deep zonal currents in the central equatorial Pacific. J. Mar. Res., 45, 791-812.

Fruman, M., B. Hua, and R. Schopp. 2009. Equatorial zonal jet formation through the barotropic instability of a low-frequency mixed Rossby gravity wave, equilibration by inertial instability, and transition to super-rotation. J. Atmos. Sci. (in press) available at http://www.geo.unifrankfurt.de/iau/ThMet/Mitarbeiter/Fruman/fruman_hua_schopp_jas_2009_jets.pdf.

Gill. 1974. The stability of planetary waves on an infinite beta-plane. Geophys. Fluid Dyn., 6, 29-47.

Gouriou, Y., B. Bourlès, H. Mercier, and R. Chuchla. 1999. Deep jets in the equatorial Atlantic Ocean. J. Geophys. Res., 104, 21216-21226.

Gouriou, Y., C. Andrié, B. Bourlès, S. Freudenthal, S. Arnault, A. Aman, G. Eldin, Y. du Penhoat, F. Baurand, F. Gallois, and R. Chuchla. 2001. Deep circulation in the equatorial Atlantic Ocean. Geophys. Res. Letts., 28(5), 819-822.

Hua, B. L., M. d’Orgeville, M. D. Fruman, C. Ménesguen, R. Schopp, P. Klein, and H. Sasaki. 2008. Destabilization of mixed Rossby gravity waves and equatorial zonal jets formation. J. Fluid Mech., 610, 311-341.

Johnson, G. C. and D. Zhang. 2003. Structure of the Atlantic ocean equatorial deep jets. J. Phys. Oceanogr., 33, 600-609.

Kawase, M., M. Rothstein, L., and R. Springer, S. 1992. Encounter of a deep western boundary current with the equator: A numerical spin-up experiment. J. Geophys. Res., 97, 5447-5463.

Lacasce, J. H. and J. Pedlosky. 2004. The instability of Rossby basin modes and the oceanic eddy field. J. Phys. Oceanogr., 34, 2027-2041.

Leetma, A. and P. F. Spain. 1981. Results from a velocity transect along the equator from $125^{\circ} w$ to $159^{\circ} w$. J. Phys. Oceanogr., 11, 1030-1033.

Luyten, J. R. and J. C. Swallow. 1976. Equatorial undercurrents. Deep-Sea Res., 23, 999-1001. 
Manfroi, A. J. and W. R. Young. 1999. Slow evolution of zonal jets on the beta-plane. J. Atmos. Sci., 56, 784-800.

Maximenko, N., A., B. Bang, and H. Sasaki. 2005. Observational evidence of alternating zonal jets in the world. Geophys. Res. Letts., 32 .

Ménesguen, C., B. L. Hua, M. D. Fruman, and R. Schopp. 2009. Intermittent mixing in equatorial deep jets. submitted.

Muench, J., E. Kunze, and E. Firing. 1994. The potential vorticity structure of equatorial deep jets. J. Phys. Oceanogr., 24, 418-428.

Ollitrault, M., M. Lankhorst, D. Fratantoni, P. Richardson, and W. Zank. 2006. Zonal intermediate currents in the equatorial Atlantic ocean. Geophys. Res. Letts., 33 .

Rhines, P. 1975. Waves and turbulence on a beta-plane. J. Fluid Mech., 69, $417-443$.

Richards, K., N. Maximenko, F. Bryan, and H. Sasaki. 2006. Zonal jets in the Pacific. Geoph. Res. Let.

Thompson, A. 2006. Eddy fluxes in baroclinic turbulence. $\mathrm{PhD}$ thesis, University of California, San Diego.

Vallis, G. and M. Maltrud. 1993. Generation of mean flows and jets on a beta plane and over topography. J. Phys. Oceanogr., 23, 1346-1362.

Weisberg, R. H. and A. M. Horigan. 1981. Low-frequency variabilityin the equatorial Atlantic. J. Phys. Oceanogr., 11, 913-920. 